\title{
17. RADIOLARIANS FROM THE DISTAL BENGAL FAN IN THE EQUATORIAL INDIAN OCEAN1
}

\author{
Kozo Takahashi
}

\begin{abstract}
Cores recovered from three sites of Leg 116 were studied for radiolarians. Generally, radiolarians were absent from most samples prepared for examination. Moderate to well-preserved radiolarian assemblages are found only in the uppermost one or two cores that were the focus of this study. All of the radiolarian assemblages in the upper cores belong to the Buccinosphaera invaginata Zone of latest Quaternary age. However, there is one stratum where a few Miocene radiolarians are reworked into the modern assemblages. Local seamounts are suggested sources for the reworked radiolarians.
\end{abstract}

\section{INTRODUCTION}

On ODP Leg 116 we drilled three sites in the distal Bengal Fan, just south of the Equator in the eastern Indian Ocean (Fig. 1). The three closely spaced sites are at the distal end of the Bengal Fan, where sediment thickness is approximately $1.5 \mathrm{~km}$ or slightly less (Cochran, Stow, et al., 1989). Sites 717, 718 , and 719 were drilled $828 \mathrm{~m}, 960 \mathrm{~m}$, and $466 \mathrm{~m}$ below the seafloor, respectively. Turbidites, devoid of useful radiolarians, comprised the major portion of bulk sediments drilled at all sites (Stow, Cochran, et al., 1989). In addition to corecatcher samples studied on board JOIDES Resolution, generally two samples from each core were examined for presence of radiolarians. Moderate to well-preserved radiolarian assemblages were found only in the upper one or two cores and thus the focus of this study was on these intervals. For this reason, the upper two cores at both Holes 717B and 719A were more thoroughly studied than others.

\section{METHODS}

Radiolarian sample preparation technique follows that of Sanfilippo et al. (1985), and species identification follows those of Nigrini and Moore (1979), Takahashi and Honjo (1981), Sanfilippo et al. (1985), Caulet (1988), and Nigrini (1988). The prepared sample slides were first scanned to determine relative number (abundant, common, rare, trace, and absent) of radiolarians present. Those that contained "abundant, common, or rare" radiolarians were further subjected to detailed species identification and counting. In the semi-quantitative census of the species, three categories of relative abundance of each species counted were assigned: abundant (A), common (C), and rare (R).

\section{RESULTS}

Examination of an additional two samples from each core yielded little additional stratigraphic information at each of the sites below uppermost cores 1 and 2. This is due to virtual absence of radiolarians throughout cores except as mentioned below. Detailed descriptions of samples from these sections of the cores (total of 248) are presented elsewhere (Cochran, Stow, et al., 1989).

\footnotetext{
${ }^{1}$ Cochran, J. R., Stow, D.A.V., et al., 1990. Proc. ODP, Sci. Results, 116: College Station, TX, U.S.A. (Ocean Drilling Program).

2 Department of Geology and Geophysics, Woods Hole Oceanographic Institution, Woods Hole, MA 02543.
}

The intervals where radiolarians are found below the uppermost cores are listed (Table 1). Radiolarian specimens are fragmented and often pyritized, and species identifications are very difficult because transmitted light does not penetrate pyrite. Scanning electron microscopy did not improve identification much because of the large proportion of broken skeletons.

The relative abundance of radiolarians in the uppermost cores is illustrated (Fig. 2). The sequence of the radiolarian abundance strata from each hole is rather similar and they are underlain by characteristic turbidite sequences directly below. The assemblages that were determined to contain "abundant, common, or rare" radiolarians were further studied for species analysis; the results are presented in Table 2. The assemblages in these cores are almost predictably similar to one another, except for two samples that contained Miocene reworked radiolarians (Table 2). The "abundant" assemblages usually contain 30 to 50 taxa of very similar constituents, whereas the "common" assemblages contain approximately 10 to 20 taxa. Almost as a rule, the "rare" assemblages contain only a few taxa, including Acanthodesmia vinculata, Tholospyris sp. group and Tetrapyle octacantha (Table 2). The constituents of the "abundant" samples are very similar to biocoenosis (Takahashi and Honjo, 1981), suggesting a modern component of the assemblages. The assemblages contained in the upper cores belong to the Buccinosphaera invaginata Zone, extending from $0.21 \mathrm{~m} . \mathrm{y} . \mathrm{BP}$ to the present (Knoll and Johnson, 1975), of the uppermost Quaternary. However, there is one zone where Miocene radiolarians had been reworked and contributed a minor part of the modern assemblages. The Miocene assemblage belongs to the Diartus petterssoni Zone, 13 to 8.5 m.y.BP (Riedel and Sanfilippo, 1978; Berggren, 1985), middle to late Miocene. The assemblage in two slides of Core 116-717B-1-CC contains a total of more than 50 specimens of Stichocorys delmontensis as well as five to ten specimens of Diartus petterssoni and Didymocyrtis laticonus.

\section{DISCUSSION}

\section{Radiolarian Preservation}

The apparent lack of radiolarian preservation in most cores from Sites 717,718 , and 719 below the uppermost cores is mainly attributed to turbidites. As clearly documented by Cochran, Stow, et al. (1989), the bulk of sediments at all sites of Leg 116 are composed of turbidites. Very small amounts of pelagic sediments are found to be interbedded between these turbidites. This is likely due to resuspension of the pelagic sediments caused by turbidity current, which are then mixed 


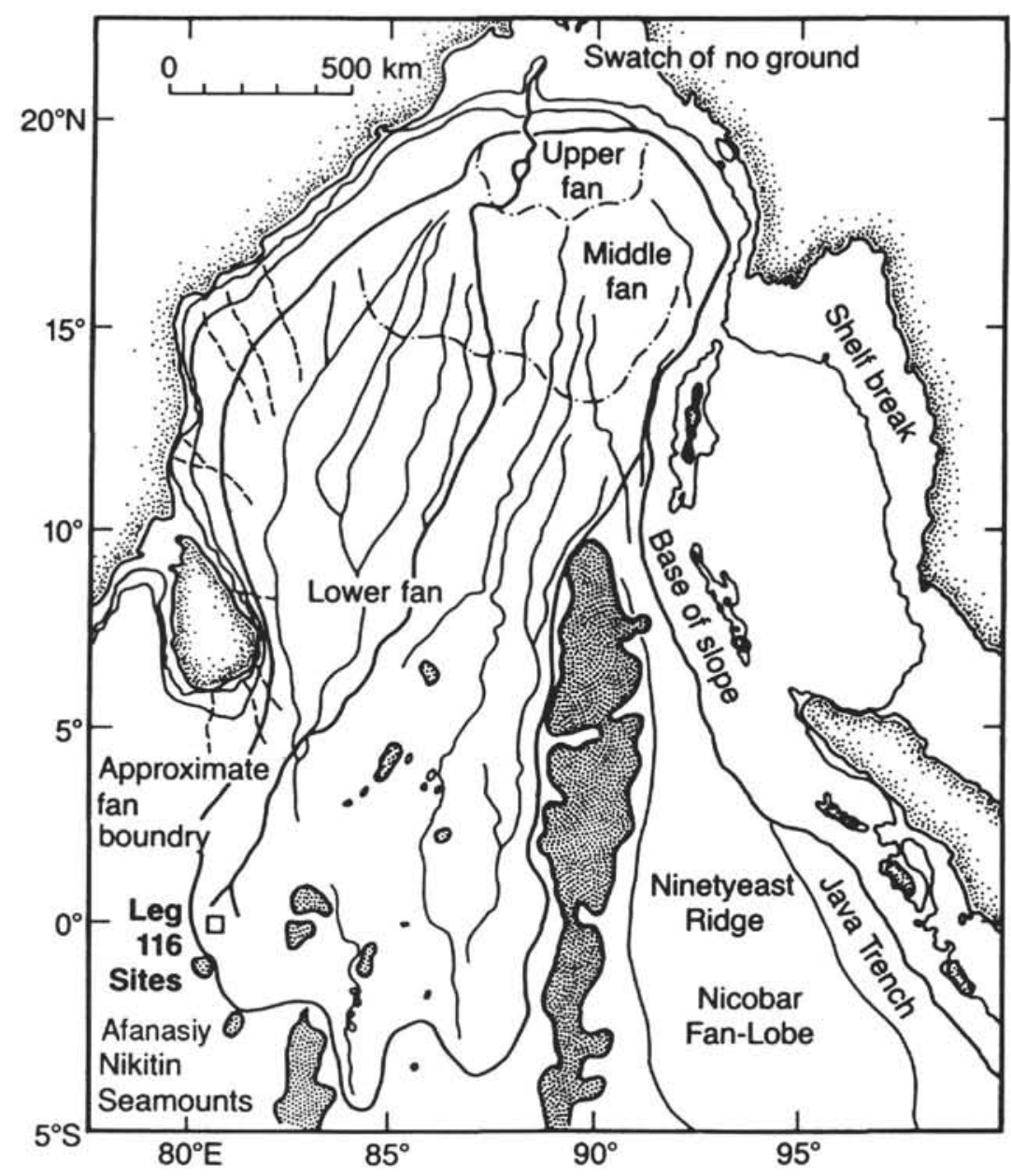

Figure 1. Map of the Bengal Fan showing the Leg 116 sites. Indian continental margin channels and fan channels are shown by dashed lines and solid lines, respectively; the currently active fan channel is shown by a heavy solid line; seamounts and topographic highs are indicated by heavy shading (after Emmel and Curray, 1984).

into the turbidite deposits. As we have documented that turbidity currents occurred about every $1000 \mathrm{yr}$ at Leg 116 sites (Stow, Cochran et al., 1989) the relative amount of pelagic sediments (perhaps on the order of $1 \mathrm{~mm} / 1000 \mathrm{yr}$ ) is small or even negligible compared to turbidite deposits a few $\mathrm{cm}$ to greater than a meter thick. Therefore, fossil radiolarians, if present, are likely diluted by coarse grains such as silt and sand and further subjected to dissolution in the highly porous interstitial waters where continuous diffusion of dissolved silicon is likely to occur. Even in pelagic clayey sediments examined (e.g., reddish brown clay, Core 116718C-94X), generally no radiolarians are present except for the uppermost cores.

Interpretation of the radiolarian record as post-depositional loss of radiolarians rather than low biosiliceous production during the Neogene in the tropical Indian Ocean is preferred. Although there is some evidence suggesting biosiliceous production in the low-latitude Indian Ocean during a part of the Neogene was less than in other oceans, the evidence still does not explain the virtual absence of radiolarians throughout the Neogene. Radiolarians from Leg 115, western Indian Ocean, are found to be scarce throughout Oligocene to middle Miocene, but common and well preserved radiolarians are found during late Miocene through Holocene (Johnson, in press). Therefore, we hypothe- size that the turbidites are responsible for the almost complete lack of preserved radiolarians throughout the cores.

\section{Reworked Radiolarians}

The reworked radiolarians found in the Samples 116-717B$1 \mathrm{H}-\mathrm{CC}$ and $-719 \mathrm{~A}-1 \mathrm{H}-2,110-111 \mathrm{~cm}$, can be confined to the D. petterssoni Zone, middle to late Miocene. As we found no other radiolarians of other ages, we think that there was only a single source for the reworked sediments. The most probable source is nearby seamounts such as Afanasiy Nikitin Seamounts (Fig. 1). At the source location, original pelagic sedimentation without turbidite disturbance occurred during the middle to late Miocene and then this particular stratum was consequently eroded to supply the reworked radiolarians to Leg 116 sites between 0.21 m.y.BP to the present.

\section{ACKNOWLEDGMENTS}

This paper was critically reviewed by David A. Johnson and an anonymous reviewer. The National Science Foundation and the Joint Oceanographic Institutions, Inc. are thanked for the sponsorship of the Ocean Drilling Program through the Texas A\&M Research Foundation under which this study was conducted. This is Contribution No. 7193 of the Woods Hole Oceanographic Institution. 
Table 1. List of samples where radiolarians were found, except for the uppermost cores listed in Figure 2. Most of these samples contain only broken and pyritized radiolarian specimens except for the samples with asterisks.

\begin{tabular}{clc}
\hline $\begin{array}{c}\text { Hole 717C } \\
\text { Core, section } \\
\text { interval }(\mathrm{cm})\end{array}$ & $\begin{array}{c}\text { Hole 718B } \\
\text { Core, section } \\
\text { interval }(\mathrm{cm})\end{array}$ & $\begin{array}{c}\text { Hole 719A } \\
\text { Core, section } \\
\text { interval }(\mathrm{cm})\end{array}$ \\
\hline 21X-CC & 4X-CC & $31 \mathrm{X}-3,113-115$ \\
26X-CC & $10 \mathrm{X}-\mathrm{CC}$ & $31 \mathrm{X}-\mathrm{CC}$ \\
27X-1, 24-26 & 11X-1, 2-4 & $38 \mathrm{X}-5,78-80^{\mathrm{c}}$ \\
$27 \mathrm{X}-5,12-14$ & $41 \mathrm{X}-3,20-26^{\mathrm{b}}$ & \\
$32-34$ & & \\
$70-72$ & & \\
$91-95$ & & \\
$116-118$ & & \\
$29 \mathrm{X}-\mathrm{CC}$ & \\
$59 \mathrm{X}-2,34-36$ & & \\
$4,11-13$ & & \\
\hline
\end{tabular}

${ }^{\text {a }}$ Moderately diverse assemblage including B. scutum, $C$. profunda, $T$. octacantha, C. papillosum, Spirocyrtis cf. subscaris, Carpocanarium sp. D. Theocaryptra sp. and Spongodiscids.

${ }^{b}$ Contains three specimens of Acrosphaera spinosa fasciculopora or $A$. spinosa hamospinosa; subspecies identification not possible due to broken tips of their spines. These two subspecies are abundant near the end of Stichocorys peregrina Zone of lower Pliocene (Caulet, 1986).

' One specimen each of Anthcyrtidium ehrenbergi ehrenbergi and Lamprocyrtis sp. were found. A. $e$. ehrenbengi is a Miocene species.

\section{REFERENCES}

Berggren, W. A., Kent, D. V., Flynn, J. J., and Van Couvering, J. A., 1985. Cenozoic geochronology. Geol. Soc. Am. Bull., 96:1407-1418.

Caulet, J. P., 1988. The genus Anthocyrtidium (Radiolaria) from the tropical late Neogene of the Indian and Pacific Oceans. Micropaleontology, 34:341-360.

Cochran, J. R., Stow, D.A.V., et al., 1989. Proc. ODP, Init. Repts., 116: College Station, TX (Ocean Drilling Program).

Emmel, F. J., and Curray, J. R., 1984. The Bengal submarine fan, northeastern Indian Ocean. Geo-Mar. Lett., 3:119-124.

Johnson, D. A., in press. Radiolarian biostratigraphy in the central Indian Ocean, Ocean Drilling Program Leg 115. In Backman, J., Duncan, R. A., et al., 1989. Proc. ODP, Sci. Results, 115: College Station, TX (Ocean Drilling Program).

Knoll, A., and Johnson, D. A., 1975. Late Pleistocene evolution of the collosphaerid radiolarian Buccinosphaera invaginata Haeckel. Micropaleontology, 21:60-68.

Nigrini, C., 1988. The genus Pterocorys (Radiolaria) from the tropical late Neogene of the Indian and Pacific oceans. Micropaleontology, $34: 217-235$.

Nigrini, C., and Moore, T. C., 1979. A guide to modern radiolaria. Spec. Publ. Cushman Found. Foraminiferal Res., 16:i-xii.

Riedel, W. R., and Sanfilippo, A., 1978. Stratigraphy and evolution of tropical Cenozoic radiolarians. Micropaleontology, 24:61-96.

Sanfilippo, A., Westberg-Smith, M. J., and Riedel, W. R., 1985. Cenozoic radiolaria. In Bolli, H. M., Saunders, J. B., and PerchNielsen, K. (Eds.), Plankton Stratigraphy: Cambridge (Cambridge Univ. Press), 631-712.

Stow, D.A.V., Cochran, J. R., and ODP Leg 116 Shipboard Scientific Party, 1989. The Bengal Fan: some preliminary results from ODP drilling. Geo-Mar. Lett., 9:1-10.

Takahashi, K., and Honjo, S., 1981. Vertical flux of Radiolaria: a taxon-quantitative sediment trap study from the western tropical Atlantic. Micropaleontology, 27:140-190.

Date of initial receipt: 11 April 1989

Date of acceptance: 2 October 1989

Ms 116B-123 


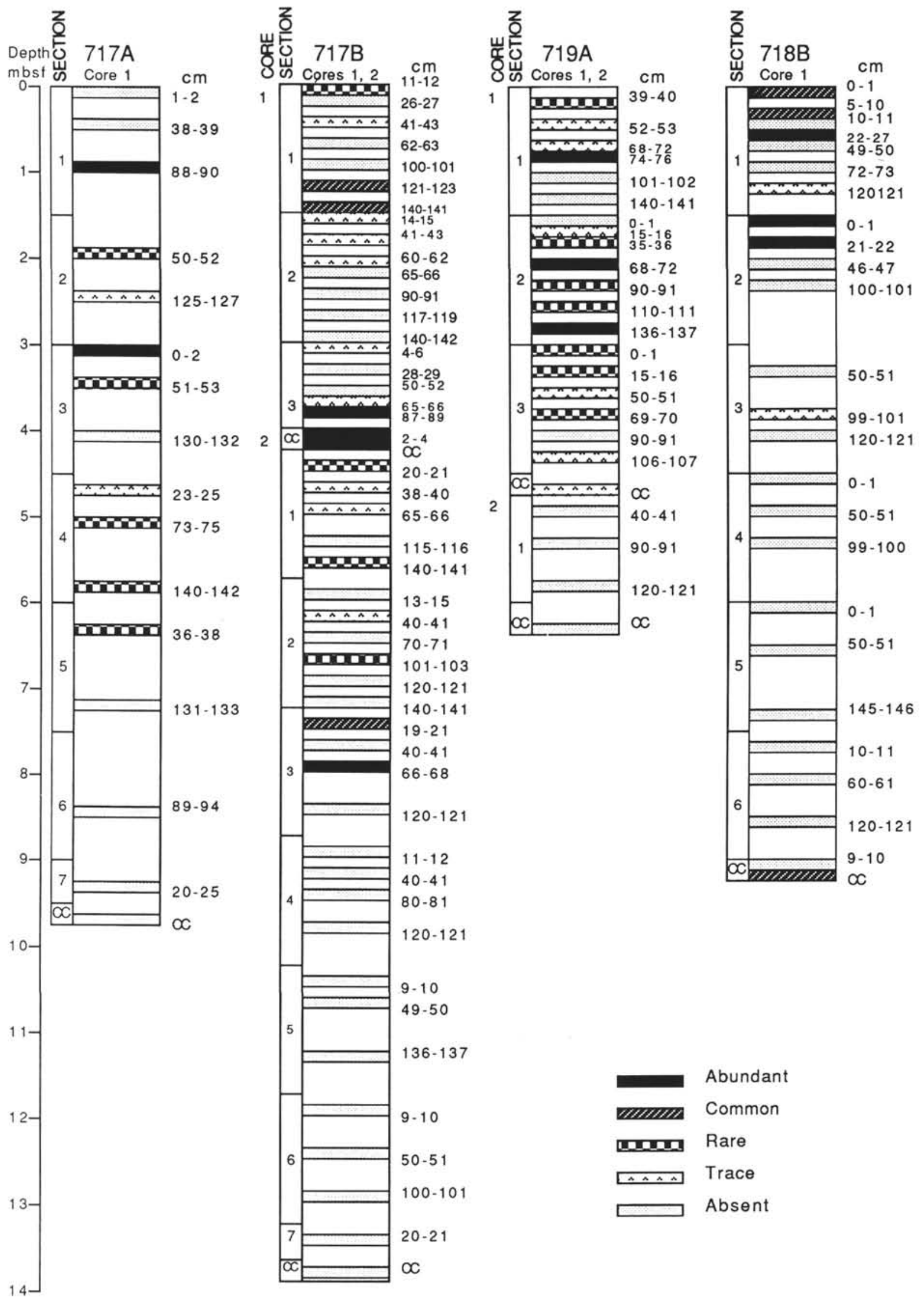

Figure 2. Relative abundance of whole radiolarians in the uppermost cores from three sites of Leg 116. Five abundance categories are employed. 
Table 2. Relative abundance of radiolarian species from uppermost cores at Holes 717A, 717B, 718B, and 719A of Leg 116. Only the samples containing abundant, common, and rare radiolarians listed in Figure 2 were examined for abundance of each species and listed here. The following designations are employed for relative abundance of each species: A: abundant; C: common; R: rare; and blank for absent.

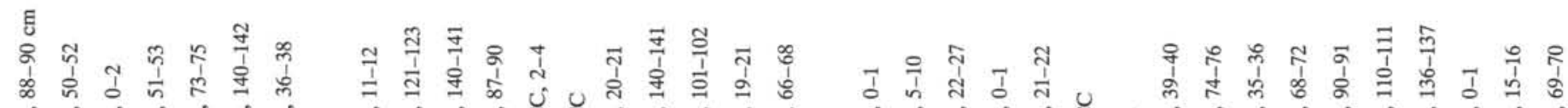

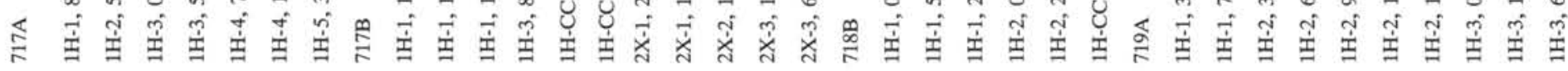

SPUMELLARIA

Acrosphaera murrayana Acrosphera spinosa

Amphirhopalum ypsilon

Buccinosphaera invaginata

Collosphaera macropora

Collosphaera polygona

Collosphaera tuberosa

Collosphaera huxleyi

Dictyocoryne truncatum

Dictyocoryne profunda

Didymocyrtis t. tetrathalamus

Disolenia zanguebarica

Druppatractus acquilonius

Euchitonia elegans

Heliodiscus asteriscus

Hexacontium axotrias

Otosphaera polymorpha

Porodiscus spp.

Saturnalis circularis

Siphonosphaera

Stylosphaera group

Tetrapyle octacantha

\section{NASSELLARIA}

Acanthodesmia vinculata

Anthocyrtidium ophirense

Anthocyrtidium zanguebaricum

Artostrobus annulatus

Botryocyrtis scutum

Botryostrobus aquilonaris

Carpocanistrum cephalum

Carpocanarium papillosum

Centrobotrys thermophila

Clathrocanium diadema

Cormutella profunda

Dictyophimus infabricatas

Eucecryphalus sestrodiscu

Eucyrtidium acuminatum

Eucyrtidium hexagonatum

Lamprocyclas maritalis maritalis

Lamprocyclas maritalis polypor

Lamprocyrtis nigriniae

Lamprocyrtis hannai

Liniospyris reticulat

Lithopera bacca

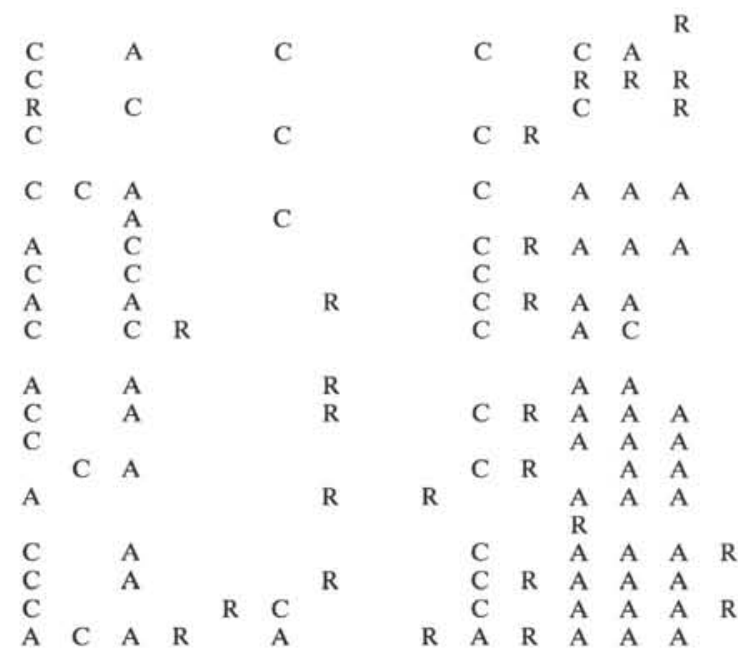

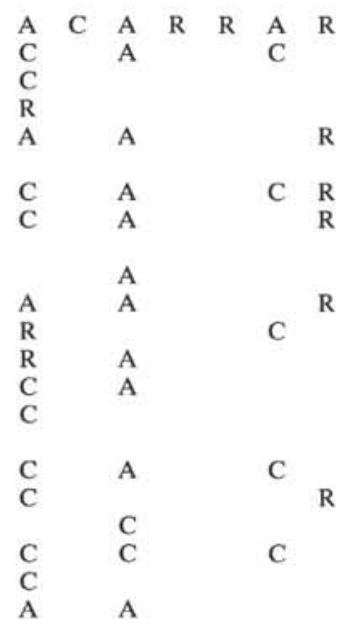

$\begin{array}{lllll}C & \mathrm{R} & \mathrm{A} & \mathrm{A} & \mathrm{A} \\ \mathrm{C} & & \end{array}$

$\mathrm{R}$

$\begin{array}{lll}\text { A } & \text { A } & \text { A } \\ & \text { A } & \text { A }\end{array}$

$\begin{array}{lll}\mathrm{R} & & \\ \mathrm{C} & \mathrm{A} & \mathrm{A}\end{array}$

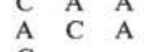

A A A

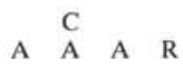

$\begin{array}{lll}\mathrm{R} & & \mathrm{R} \\ \mathrm{A} & \mathrm{A} & \end{array}$

$\begin{array}{cc}\text { C } & \text { A } \\ & \text { A } \\ & \text { A } \\ \text { A } & \text { A } \\ \text { C } & \text { A }\end{array}$



$\begin{array}{llllll} & & A & & A & \\ C & R & A & A & A & R\end{array}$

$\begin{array}{lllll}\text { C } & \text { A } & \text { A } & \text { A } & \text { R } \\ \text { C } & \text { C } & & \text { A } & \end{array}$

A A A

$\begin{array}{rll} & \mathrm{C} & \mathrm{A} \\ \mathrm{A} & \mathrm{A} \\ \mathrm{A} & \mathrm{A}\end{array}$

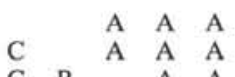

$\begin{array}{llllll}\text { C } & \mathrm{R} & & \mathrm{A} & \mathrm{A} & \mathrm{R} \\ \mathrm{C} & \mathrm{R} & \mathrm{A} & \mathrm{A} & \mathrm{A} & \mathrm{R}\end{array}$

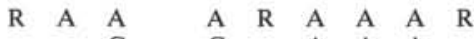

$\begin{array}{lll}\mathrm{A} & \mathrm{A} & \mathrm{A} \\ \mathrm{C} & \mathrm{C} & \end{array}$

A A $\begin{array}{r}\text { R } \\ \text { A }\end{array}$

$\begin{array}{llll}\text { A } & \text { A } & \text { A } & \text { R } \\ \text { C } & \text { A } & & \end{array}$

C A $\mathrm{R}$
A A A

A C A

A $\quad$ A

$\begin{array}{lll}\mathrm{C} & \mathrm{C} & \mathrm{A} \\ \mathrm{C} & & \mathrm{A}\end{array}$

C $\stackrel{\mathrm{R}}{\mathrm{C}} \mathrm{C}$

C $\quad \begin{array}{lll}\text { A } & \text { A } & \text { A } \\ & \text { A } & \text { C }\end{array}$

A $\stackrel{A}{A}$
$\begin{array}{llll}\mathrm{R} & \mathrm{C} & \mathrm{A} & \mathrm{A}\end{array}$

$\begin{array}{lll}\mathrm{R} & \mathrm{C} & \mathrm{A} \\ & \mathrm{C} & \end{array}$

C C A

$\begin{array}{lllll}\text { R } & \text { C } & \text { A } & \text { A } & \text { R } \\ \text { C } & \text { A } & & \end{array}$

A

$\begin{array}{llllll} & & & \text { C } & & \text { C } \\ \text { C } & & \text { A } & & \\ \text { C } & & & \text { R } & \text { C } \\ \text { A } & & \text { A } & & \end{array}$ 


\begin{tabular}{|c|c|c|c|c|c|c|c|c|c|c|c|c|c|c|c|c|c|c|c|c|c|c|c|c|c|c|c|c|c|c|c|c|c|c|c|}
\hline & $\stackrel{\nwarrow}{ミ}$ & 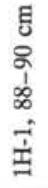 & $\begin{array}{l}\text { กี } \\
1 \\
0 \\
\text { กิ } \\
\text { İ }\end{array}$ & $\begin{array}{l}\text { Tे } \\
\vdots \\
\hat{3} \\
\stackrel{I}{I}\end{array}$ & $\begin{array}{l}\hat{n} \\
\frac{1}{n} \\
\dot{n} \\
\dot{1}\end{array}$ & $\begin{array}{l}\frac{n}{1} \\
\frac{n}{2} \\
\dot{J} \\
\pm\end{array}$ & 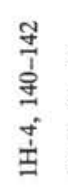 & 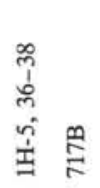 & $\begin{array}{l}I \\
I \\
\pm \\
I\end{array}$ & 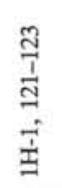 & $\begin{array}{l}\frac{F}{J} \\
\frac{1}{a} \\
\vec{I} \\
\dot{I}\end{array}$ & $\begin{array}{l}8 \\
\frac{1}{1} \\
\infty \\
⿱ ㇒ \\
1 \\
1\end{array}$ & 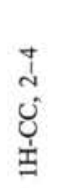 & $\begin{array}{l}\text { U } \\
\text { II }\end{array}$ & 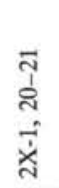 & 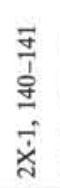 & 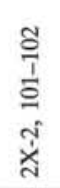 & $\begin{array}{l}\vec{T} \\
\stackrel{a}{a} \\
\dot{3} \\
\dot{d}\end{array}$ & 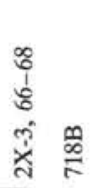 & $\begin{array}{l}I \\
I \\
I\end{array}$ & $\begin{array}{l}9 \\
1 \\
n \\
\vdots \\
\pm\end{array}$ & $\begin{array}{l}\tilde{A} \\
\text { İ } \\
\dot{I} \\
I\end{array}$ & $\begin{array}{l}7 \\
1 \\
0 \\
1 \\
7\end{array}$ & $\begin{array}{l}\text { ปี } \\
\frac{1}{N} \\
\hat{N} \\
\frac{I}{I}\end{array}$ &  & \& & $\begin{array}{l}\frac{I}{I} \\
\frac{1}{I}\end{array}$ & 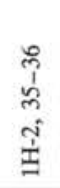 & 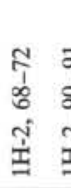 &  & 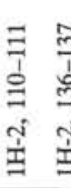 & 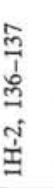 & $\begin{array}{l}7 \\
0 \\
\text { m } \\
\stackrel{1}{I}\end{array}$ & 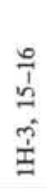 & $\begin{array}{l}\text { p. } \\
\text { ó } \\
\text { m. } \\
\dot{I}\end{array}$ \\
\hline Lophospyris pentagona hyperborea & & C & & & & & & $\mathbf{R}$ & & & & c & A & A & & & & & & & & A & $\mathrm{C}$ & & $\mathrm{R}$ & & & & A & & A & & & & \\
\hline Lophospyris pentagona pentagona & & $\mathrm{C}$ & & & & & & & & & & $\mathrm{C}$ & & & & & & & $\mathrm{C}$ & & & & & & $\mathrm{R}$ & & & & & & C & & & & \\
\hline Peripyramis circumtexta & & C & & & & & & & & & & & C & $\mathrm{R}$ & & & & & & $\mathrm{C}$ & & & & $\mathrm{C}$ & & & $\mathrm{C}$ & & & & C & & & & \\
\hline Peromelissa pharacra & & A & & & & & & & $\mathrm{R}$ & A & & A & A & & & $\mathbf{R}$ & & & A & C & & A & A & & & & & & A & & C & & & & \\
\hline Phormostichoartus corbula & & $\mathrm{C}$ & & A & & & & $\mathbf{R}$ & & & & & A & A & & & & & $\mathrm{C}$ & & & $\mathrm{C}$ & A & $\mathrm{C}$ & & & $\mathrm{C}$ & & A & & A & & & & \\
\hline Pterocanium p. praetextum & & A & $\mathrm{C}$ & A & & & & & $\mathrm{R}$ & & $\mathrm{R}$ & A & A & & & & & & A & $\mathrm{C}$ & & A & A & A & & & A & & A & & A $\mathrm{R}$ & $\mathrm{R}$ & & & \\
\hline Pterocanium trilobum & & $\mathrm{C}$ & & $\mathrm{C}$ & & & C & & & & & A & A & A & & & & $\mathrm{C}$ & A & $\mathrm{C}$ & & A & A & A & & & & & A & & & & & & \\
\hline Pterocorys macroceras & & $\mathrm{R}$ & & & & & & & & & & & & & & & & & & & & & & & & & & & & & & & & & \\
\hline Pterocorys hertwigii & & C & & & & & & & & & & & & & & & & & & & & & & & & & & & & & & & & & \\
\hline Pterocorys sabae & & A & $\mathrm{C}$ & A & & & & & & $\mathrm{C}$ & & A & A & A & & & & & A & $\mathrm{C}$ & & A & A & A & & & A & & A & & A $\mathrm{R}$ & $\mathbf{R}$ & & & \\
\hline Pterocorys clausus & & & & $\mathrm{R}$ & & & & & & & & & & & & & & & & & & C & & $\mathrm{C}$ & & & & & & & C & & & & \\
\hline Siphocampe arachnea & & $\mathrm{C}$ & & $\mathrm{C}$ & & & & $\mathbf{R}$ & & & & A & $\mathrm{C}$ & & & & & A & C & & & $\mathrm{C}$ & $\mathrm{C}$ & & & & & & A & & & & & & \\
\hline Siphocampe lineata & & $\mathrm{C}$ & & A & & & & $\mathrm{R}$ & $\mathrm{R}$ & & & A & A & & & & & & $\mathrm{C}$ & & & A & $\mathrm{C}$ & & & & & & $\mathrm{C}$ & & & & & & \\
\hline Spirocyrtis cf. subscalaris & & $\mathrm{C}$ & $\mathrm{C}$ & A & & & & $\ddot{R}$ & & $\mathrm{C}$ & & & A & A & & & & & A & & & A & & A & & & & & A & & $\mathrm{C}$ & & & & \\
\hline Theocalyptra sp. & & & & A & & & & & & & & $\mathrm{C}$ & $\mathrm{C}$ & & & & & & C & & & A & C & C & & & & & & & $\mathrm{C}$ & & & & \\
\hline Theocorys veneris & & $\mathrm{C}$ & & $\mathrm{A}$ & & & & & & & & $\mathrm{C}$ & $\mathrm{C}$ & & & & & & & & & $\mathrm{C}$ & & C & & & & & C & & & & & & \\
\hline Theocorythium $t$. trachelium & & $\mathrm{C}$ & & A & & & & & & & & A & A & A & & & & & $\mathrm{C}$ & $\mathrm{C}$ & & A & A & A & & & $\mathrm{C}$ & & $\mathrm{C}$ & & & & & & \\
\hline Tholospyris sp. group & & A & $\mathrm{C}$ & A & $\mathrm{R}$ & $\mathrm{R}$ & A & & $\mathrm{R}$ & A & & A & A & & $\mathrm{R}$ & $\mathrm{R}$ & $\mathrm{R}$ & A & A & A & $\mathrm{C}$ & A & A & & $\mathrm{R}$ & R & & $\mathrm{R}$ & A & & A $\mathrm{R}$ & $\mathbf{R}$ & $\mathrm{R}$ & $\mathrm{R}$ & $\mathbf{R}$ \\
\hline \multicolumn{35}{|l|}{ REWORKED RADIOLARIANS } & \\
\hline Anthocyrtidium ehrenbergi & & & & & & & & & & & & & & $\mathrm{R}$ & & & & & & & & & & & & & & & & & & & & & \\
\hline Diartus petterssoni & & & & & & & & & & & & & & $\mathbf{R}$ & & & & & & & & & & & & & & & & & & & & & \\
\hline Didymocyrtis laticonus & & & & & & & & & & & & & & $\ddot{R}$ & & & & & & & & & & & & & & & & & & & & & \\
\hline Lychnodictyum audax & & & & & & & & & & & & & & $\hat{R}$ & & & & & & & & & & & & & & & & & & & & & \\
\hline Siphostichartus corona & & & & & & & & & & & & & & R & & & & & & & & & & & & & & & & & $\mathbf{R}$ & & & & \\
\hline Stichocorys delmontensis & & & & & & & & & & & & & & $\mathrm{C}$ & & & & & & & & & & & & & & & & & $\mathrm{C}$ & & & & \\
\hline
\end{tabular}

